Article original

\title{
Lien entre le niveau de lipolyse du lait de chèvre et la qualité sensorielle des fromages au lait cru ou pasteurisé
}

\author{
François MORGAN ${ }^{\mathrm{a}}$, Jean-Pierre BODIN ${ }^{\mathrm{b}}$, Patrice GABORIT ${ }^{\mathrm{a} *}$ \\ ${ }^{a}$ ITPLC, Institut Technique des Produits Laitiers Caprins, BP 49, 17700 Surgères, France \\ b ENILIA, École Nationale d'Industrie Laitière et des Industries Agroalimentaires, \\ 17700 Surgères, France
}

(Reçu le 15 janvier 2001 ; accepté le 9 avril 2001)

\begin{abstract}
Link between goat milk lipolysis and sensorial quality of lactic goat cheeses made from raw or pasteurised milk. There is an important variability of the lipolysis level in goat milk, due to various physiological and breeding factors. The aim of this study was to evaluate the impact of these natural variations on the sensorial quality of fresh and ripened lactic goat cheeses. The effect of milk pasteurisation was also studied.

The results show that the variation of the lipolysis level of goat milk do not have any significant effect on the sensorial quality of ripened cheeses. On the contrary, the sensorial quality of fresh cheeses is linked to the lipolysis level of milk.

These results allowed us to recommend some means of controlling the sensorial quality of goat cheeses. For ripened cheeses, a satisfying sensorial quality could be obtained via the control of the technological parameters, notably the moisture in non-fat cheese at the demoulding. For fresh cheeses, the lipolysis level of milk should be checked before cheesemaking, and milks with high lipolysis levels should not be used. Pasteurisation of milk induced a decrease in the lipolysis level of cheeses. The use of pasteurisation led to a limitation of the appearance of organoleptic defects in ripened cheeses, but the overall flavour and odour of ripened cheeses made from pasteurised milk was also reduced compared to cheeses made from raw milk.
\end{abstract}

goat milk / lipolysis / goat cheese / sensorial quality / pasteurisation

Résumé - Il existe une importante variabilité du niveau de lipolyse du lait de chèvre en raison de divers facteurs liés à la physiologie de l'animal et aux conditions de production. L'objectif de cette étude était d'évaluer l'impact de ces variations naturelles sur la qualité sensorielle de fromages de chèvre lactiques (type bûchette) frais et affinés. L'incidence de la pasteurisation a également été étudiée.

* Correspondance et tirés-à-part

Tél. : (33) 546276980 ; fax : (33) 546276989 ; e-mail : patrice.gaborit@itplc.asso.fr 
Les résultats montrent que le niveau de lipolyse du lait n'a pas d'effet significatif sur la qualité sensorielle des fromages affinés. En revanche, la qualité sensorielle des fromages frais est directement liée au niveau de lipolyse des laits.

Ces résultats permettent d'envisager des actions de maîtrise de la qualité sensorielle des fromages de chèvre. Pour les fromages affinés, l'obtention d'une qualité sensorielle satisfaisante nécessite une maîtrise des paramètres technologiques et notamment le contrôle de l'humidité du fromage dégraissé au démoulage. Pour les fromages frais, un contrôle du niveau de lipolyse des laits mis en fabrication doit être réalisé et l'utilisation de laits présentant des niveaux élevés de lipolyse doit être évitée. L'utilisation de laits pasteurisés permet de réduire le niveau de lipolyse des fromages et la fréquence d'apparition des défauts organoleptiques pour les fromages affinés. Toutefois, la pasteurisation des laits conduit aussi à des fromages affinés ayant une flaveur globale et une odeur globale moins prononcées.

\section{lait de chèvre / lipolyse / fromage de chèvre / qualité sensorielle / pasteurisation}

\section{INTRODUCTION}

La lipolyse joue un rôle majeur dans l'élaboration de la qualité sensorielle des fromages de chèvre lactiques [27]. La lipolyse des fromages peut être due aux lipases du lait, de la présure, des ferments lactiques et de la flore de surface [16].

Dans le lait de chèvre, la lipolyse est principalement corrélée à l'activité de la lipoprotéine lipase ou LPL [2], mais les bactéries psychrotrophes et les cellules somatiques possèdent également des activités lipasiques [6].

De fortes variations saisonnières du niveau de lipolyse des laits de chèvre ont été observées, et d'autres facteurs physiologiques ou liés aux conditions de production ont été mis en évidence [5]. L'hypothèse d'un lien entre le polymorphisme génétique au locus de la caséine $\alpha_{\mathrm{S} 1}$ et les variations individuelles du niveau de lipolyse dans le lait de chèvre a également été avancée [8].

La lipolyse conduit à la libération d'acides gras libres dans le lait et les fromages. Des quantités importantes de ces acides gras libres peuvent être préjudiciables à la flaveur du lait et des produits laitiers, en entraînant l'apparition de flaveurs rance, savon, ou piquante [3, 32]. Par ailleurs, la libération de mono et diglycérides qui ac- compagne la lipolyse peut-être à l'origine d'un défaut d'amertume dans les fromages [24]. Toutefois, la flaveur caractéristique des produits laitiers caprins est due en grande partie à certains acides gras libres, notamment aux acides gras ramifiés 4ethyl-C8 et 4-methyl-C8 [19, 25] et une corrélation entre la lipolyse et la flaveur « chèvre » du lait a été montrée $[5,19,22$, $25,31]$.

Malgré le rôle prépondérant tenu par la lipolyse dans les mécanismes de formation de la flaveur des produits laitiers caprins, il n'existe que peu d'études sur ce sujet. L'homogénéisation et le report du lait à l'état réfrigéré entraînent une augmentation du niveau de lipolyse du lait de chèvre [27]. Les traitements thermiques (thermisation et pasteurisation) provoquent en revanche une diminution de l'activité lipasique des laits de chèvre $[7,21]$. Le niveau de lipolyse des fromages de chèvre augmente au cours de l'affinage [8, 17, 25], et la qualité sensorielle des fromages affinés semble liée à l'activité lipolytique de la flore de surface [17]. D'autres travaux ont montré l'influence du polymorphisme génétique de la caséine $\alpha_{\mathrm{S} 1}$ caprine sur le niveau de lipolyse des laits et la qualité sensorielle des fromages de chèvre à pâte molle $[8,30]$ et à pâte pressée [8,22]. Un cas particulier concerne les travaux de Le Mens et al. [23], qui ont 
montré que l'ajout de lipase $\mathrm{S}$ de Rhizopus arrhizus au lait de fabrication conduit à une forte augmentation du niveau de lipolyse des fromages de chèvre lactiques frais et affinés, et est à l'origine de l'apparition de défauts de flaveur (amère, piquant, rance, savon) dans ces produits. Toutefois, la relation entre la lipolyse « naturelle » des laits mis en fabrication et la qualité sensorielle des fromages de chèvres lactiques n'a pas été directement étudiée à notre connaissance.

Ainsi, l'objectif de cette étude était d'évaluer l'impact des variations naturelles du niveau de lipolyse du lait de chèvre sur la qualité sensorielle des fromages de chèvre. Des bûchettes lactiques fraîches et affinées ont été fabriquées avec des laits possédant différents niveaux de lipolyse (180 fabrications). Les fabrications étaient réalisées avec des laits crus ou pasteurisés. Les relations existant entre les caractéristiques des laits utilisés (niveau de lipolyse et autres paramètres de composition, laits crus ou pasteurisés) et les caractéristiques biochimiques et sensorielles des fromages sont discutées. Les résultats obtenus permettent d'envisager des actions de maîtrise de la qualité sensorielle des fromages de chèvre.

\section{MATÉRIEL ET MÉTHODES}

\subsection{Laits de troupeau et fabrications fromagères}

Les laits de troupeau (laits de $72 \mathrm{~h}$, soit un mélange de 6 traites) ont été prélevés au cours de deux années consécutives, dans 9 élevages de Poitou-Charentes. Les prélèvements de la première année $(n=18)$ étaient répartis sur l'ensemble de la période de lactation, ceux de la deuxième année $(n=12)$ étaient effectués en milieu de lactation (mai-juin). Le niveau de lipolyse des laits de troupeau était situé sur une large gamme allant de 0,1 à $1,9 \mathrm{~g} \mathrm{AO} \cdot 100 \mathrm{~g}^{-1}$ MG.
Les laits étaient mis en fabrication immédiatement après les prélèvements, sans nouvelle période de report au froid. Une partie du lait était pasteurisé $\left(74{ }^{\circ} \mathrm{C}, 50 \mathrm{~s}\right)$ avant d'être mis en fabrication. Pour chaque type de lait (cru ou pasteurisé), 3 lots de fromages de chèvres lactiques (format bûchettes) étaient obtenus : 1 lot de fromages frais emballés sous vide et conservés 2 semaines à $4{ }^{\circ} \mathrm{C}$ avant analyses, 1 lot de fromages affinés $10 \mathrm{j}$ à $12^{\circ} \mathrm{C}$ et conservés 4 semaines à $4{ }^{\circ} \mathrm{C}$ avant analyses (FA4), et 1 lot de fromages affinés $10 \mathrm{j}$ à $12^{\circ} \mathrm{C}$ et conservés 8 semaines à $4{ }^{\circ} \mathrm{C}$ (date limite d'utilisation optimale) avant analyses (FA8). Les fabrications fromagères étaient réalisées selon le schéma technologique décrit figure 1. Les ferments lactiques utilisés étaient un mélange de ferments mésophiles (FLORA DANICA MSP à $15 \mathrm{u} \cdot 100 \mathrm{~kg}^{-1}$ de lait et R703 à $6 \mathrm{u} \cdot 100 \mathrm{~kg}^{-1}$ de lait ; CHR Hansen, Arpajon, France). La présure utilisée était issue de la caillette de veau (présure Carlin, Texel, Dangé St Romain, France). Pour les fromages affinés, la flore de surface était composée de Geotrichum candidum (Geo 17, Texel, Dangé St Romain, France) et Penicillium candidum (Eden, Texel, Dangé St Romain, France). Les ferments lyophilisés étaient réhydratés en milieu tryptone-sel et la quantité utilisée pour la pulvérisation était de 0,7 dose $100 \mathrm{~kg}^{-1}$ de caillé pour chaque souche.

\subsection{Analyses physico-chimiques et microbiologiques}

\subsubsection{Laits}

Le niveau de lipolyse du lait, exprimé en g d'acide oléique pour $100 \mathrm{~g}$ de matière grasse (g AO.100 g $\left.\mathrm{g}^{-1} \mathrm{MG}\right)$, était déterminé par la méthode aux savons de cuivre [15].

Les teneurs en matières grasses (MG) et matières protéiques (MP) étaient déterminées par la méthode infrarouge à l'aide d'un appareil Milkoscan [13]. 


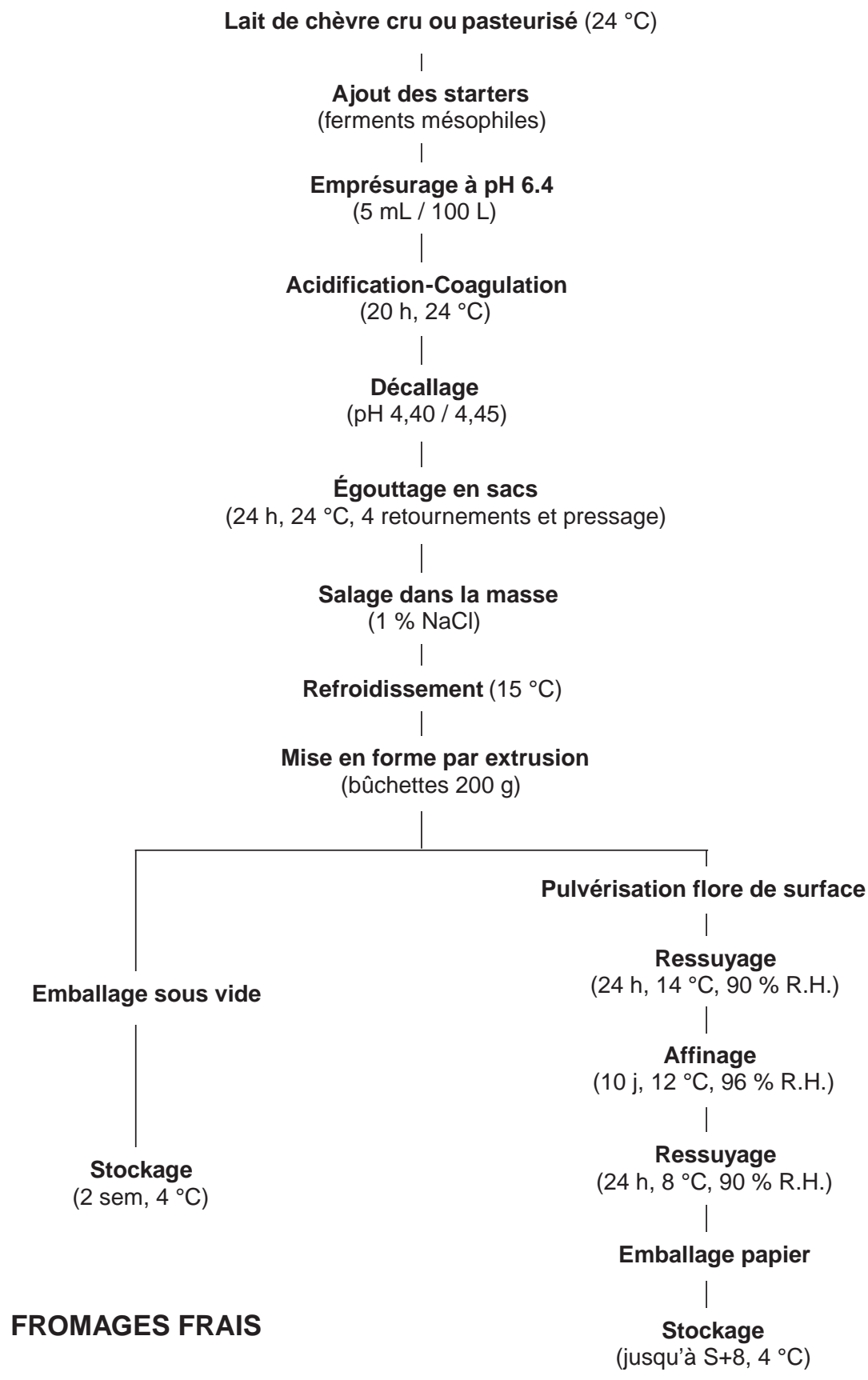

\section{FROMAGES AFFINÉS}

Figure 1. Schéma technologique pour la fabrication des fromages de chèvre lactiques. Figure 1. Technological schemes for the manufacture of lactic goat cheeses. 
Les comptages de cellules somatiques (CCS) étaient obtenus à l'aide d'un compteur Fossomatic 360 (Foss Electric, Nanterre, France), selon la norme de la FIL [12] et la flore mésophile aérobie revivifiable (FMAR) par la technique Bactoscan [15]. Les coliformes totaux [10] et les germes psychrotrophes [11] étaient dénombrés selon les méthodes de la FIL.

\subsubsection{Fromages}

Le niveau de lipolyse des fromages ( $\mathrm{g}$ AO $100 \mathrm{~g}^{-1} \mathrm{MG}$ ) était mesuré selon une procédure similaire à celle décrite par Delacroix-Buchet et al. [8]. Après acidification à $\mathrm{pH} 2$ d'un mélange sable/fromage $(50 / 50 \mathrm{~m} / \mathrm{m})$ par addition $\mathrm{d}^{\prime} \mathrm{H}_{2} \mathrm{SO}_{4}$ $4 \mathrm{~mol} \cdot \mathrm{L}^{-1}$, la matière grasse était récupérée par centrifugation $\left(3900 \mathrm{~g}, 30 \mathrm{~min}, 55^{\circ} \mathrm{C}\right.$ ) et le niveau de lipolyse était obtenu par titration selon le protocole de la FIL [14].

L'extrait sec (ES) des fromages était déterminé par pesée après dessiccation à l'étuve à $102^{\circ} \mathrm{C}$. La matière grasse des fromages était dosée selon la méthode de Heiss [20]. Ces différents dosages permettaient de calculer le rapport matière grasse sur extrait sec $(\mathrm{G} / \mathrm{S})$ et l'humidité du fromage dégraissé (HFD), exprimés en pourcentage.

\subsection{Analyse sensorielle}

Les propriétés sensorielles des fromages étaient évaluées par un groupe de 12 experts entraînés, dans des locaux spécialement aménagés [1]. Les fromages (3 fromages au lait cru et 3 fromages au lait pasteurisé par séance) étaient présentés en ordre monadique séquentiel, variant d'un expert à l'autre, en tranches cylindriques avec la croûte après élimination des talons $(2 \mathrm{~cm}$ de large, $\sim 30 \mathrm{~g})$. Les intensités des descripteurs étaient notées sur une échelle continue linéaire allant de 0 (pas de perception du descripteur) à 10 (intensité perçue très intense). L'enregistrement des intensités se faisait à l'aide du logiciel FIZZ (Biosystemes ${ }^{\circledR}$, Couternon, France). Les descripteurs évalués étaient l'odeur et la flaveur globale, la flaveur " chèvre » et 11 descripteurs de défauts les plus couramment rencontrés ( 4 défauts d'odeur, 7 défauts de flaveur). Le tableau I présente les résultats d'une étude précédente [23], au cours de laquelle un jury d'experts avait sélectionné ces descripteurs « négatifs » puis déterminé les notes moyennes correspondantes aux seuils d'apparition des défauts organoleptiques. Quand la note moyenne attribuée au descripteur dépassait cette valeur seuil, un défaut de flaveur était

Tableau I. Notes moyennes d'apparition des défauts sensoriels déterminées par le jury d'experts sur les échelles d'intensité de perception des descripteurs (D'après Le Mens et al., [22]).

Table I. Average notes for the occurrence of sensorial defects as determined by experts on the scale of perception's intensity of the descriptors (From Le Mens et al., [22]).

\begin{tabular}{lcc}
\hline Descripteurs & $\begin{array}{c}\text { Fromages } \\
\text { frais }\end{array}$ & $\begin{array}{c}\text { Fromages } \\
\text { affinés }\end{array}$ \\
\hline $\begin{array}{l}\text { Flaveur (note / 10) } \\
\text { Acide }\end{array}$ & 5,30 & \\
Amère & 1,39 & 2,40 \\
Salée & 4,31 & 4,39 \\
Métallique/oxydée & 1,13 & 1,46 \\
Piquante & 1,04 & 2,36 \\
Rance & 0,79 & 1,41 \\
Savon & 0,82 & 1,44 \\
\hline Odeur (note / 10) & & \\
Rance & 0,63 & 1,07 \\
Aigre & 1,48 & $1,48^{*}$ \\
Bleue & $2,11 *$ & 2,11 \\
Piquante & $1,98^{*}$ & 1,98 \\
\hline
\end{tabular}

* : non déterminées dans l'étude de Le Mens et al. [22]. La note utilisée pour l'apparition du défaut d'odeur aigre dans les fromages affinés est celle déterminée par les experts sur les fromages frais. Les notes utilisées pour l'apparition des défauts d'odeur bleue et piquante dans les fromages frais sont celles déterminées par les experts sur les fromages affinés. 
comptabilisé sur le produit. Selon le nombre de défauts enregistrés, les fromages était repartis dans différents groupes: Q1 (0 ou 1 défaut - qualité sensorielle satisfaisante), Q2 (2 à 5 défauts - qualité sensorielle en limite d'acceptabilité), Q3 (6 défauts et plus - qualité sensorielle inacceptable).

\subsection{Analyse statistique}

Des tests d'égalité des espérances (deux observations de variance différentes) étaient réalisés à l'aide de l'utilitaire d'analyse du logiciel Microsoft EXCEL (version 97).

\section{RÉSULTATS}

\subsection{Fromages frais}

Les caractéristiques moyennes des fromages frais de qualité Q1 (satisfaisante), Q2 (en limite d'acceptabilité) et Q3 (inacceptable) et des laits correspondants sont comparées dans le tableau II.

La catégorie Q3 contenait 8 produits sur un effectif total de 60 fromages frais. Ces produits étaient caractérisés par une flaveur et une odeur globales plus intenses que celles des fromages des catégories Q1 et Q2 (Tab. II). Aucune différence significative n'était observée concernant la flaveur chèvre des fromages des différentes catégories. De manière générale, les notes moyennes attribuées aux descripteurs de défauts étaient plus élevées pour les fromages Q3 que pour les fromages Q1 et Q2. Les principaux défauts organoleptiques des fromages frais de la catégorie Q3 étaient des odeurs rance et aigre, et des flaveurs amère, métallique/oxydée, piquante, rance et savon. Le défaut de flaveur piquant était couramment rencontré chez l'ensemble des fromages frais analysés, mais son intensité moyenne était significativement plus basse pour les fromages Q1 en comparaison aux produits Q2 et Q3 (Tab. II).

Comparés aux produits de qualité Q1 ou Q2, les fromages frais de qualité Q3 possédaient un niveau de lipolyse significativement plus élevé et un $\mathrm{G} / \mathrm{S}$ significativement plus faible (inférieur à $45 \%$ ). Ce dernier paramètre était lié à la mise en œuvre de laits présentant un ratio MG/MP inversé (Tab. II).

Le niveau de lipolyse moyen des laits utilisés pour la fabrication des fromages frais était significativement plus élevé pour les produits de qualité Q3 (Tab. II). Comme l'indique la figure 2 , une corrélation positive très hautement significative était obtenue entre le niveau de lipolyse des fromages frais et celui des laits utilisés $(r=0,95 ; p<0,001)$. Le niveau de lipolyse des fromages frais était supérieur d'un facteur 2 à 3 en moyenne à celui des laits utilisés. La figure 3 montre la corrélation entre le niveau de lipolyse des laits utilisés et le nombre de défauts rencontrés sur les fromages frais et $(r=0,85 ; p<0,001)$. Ainsi, l'utilisation de laits fortement lipolysés conduisait à l'obtention de fromages frais présentant des niveaux élevés de lipolyse et à un accroissement des défauts organoleptiques.

\subsection{Fromages affinés}

Les compositions moyennes des fromages affinés de qualité Q1 (satisfaisante), Q2 (en limite d'acceptabilité) et Q3 (inacceptable) et des laits correspondants sont comparées dans le tableau III.

La catégorie Q3 contenait seulement 5 fromages, et uniquement des fromages FA8. Ces produits étaient caractérisés par une flaveur et une odeur globales plus intenses que celles des fromages FA8 des catégories Q1 et Q2 (Tab. III). Aucune différence significative n'était observée concernant la flaveur chèvre des fromages affinés FA8 de différentes qualités 
Tableau II. Caractéristiques moyennes des fromages et des laits utilisés en fonction de la qualité sensorielle des fromages frais.

Table II. Mean characteristics of cheeses and milk used as a function of the sensorial quality of fresh cheeses.

\begin{tabular}{|c|c|c|c|}
\hline Qualité sensorielle des fromages : & $\begin{array}{c}\mathbf{Q}_{\mathbf{1}} \\
(n=13)\end{array}$ & $\begin{array}{c}\mathbf{Q}_{\mathbf{2}} \\
(n=39)\end{array}$ & $\begin{array}{c}\mathbf{Q}_{\mathbf{3}} \\
(n=8)\end{array}$ \\
\hline \multicolumn{4}{|l|}{ Caractéristiques organoleptiques * } \\
\hline Odeur globale $(/ 10)$ & 3,65 (b) & 3,70 (b) & 4,40 (a) \\
\hline Flaveur globale $(/ 10)$ & $5,53(\mathrm{~b})$ & 5,67 (b) & 6,11 (a) \\
\hline Flaveur chèvre (/10) & 3,26 & 3,41 & 3,34 \\
\hline Nombre moyen de défauts $(/ 11)$ & $0,85(\mathrm{c})$ & 2,63 (b) & 6,25 (a) \\
\hline Odeur rance $(/ 10)$ & 0,06 (b) & 0,14 (b) & 1,31 (a) \\
\hline Odeur aigre (/10) & $0,80(b)$ & 0,88 (b) & 1,57 (a) \\
\hline Odeur bleue (/10) & 0,11 (b) & 0,17 (b) & 0,66 (a) \\
\hline Odeur piquante $(/ 10)$ & 0,43 (b) & $0,51(\mathrm{ab})$ & 0,80 (a) \\
\hline Flaveur acide $(/ 10)$ & 2,98 (ab) & 3,23 (a) & $2,86(b)$ \\
\hline Flaveur amère (/10) & $0,58(\mathrm{c})$ & 0,96 (b) & 2,42 (a) \\
\hline Flaveur salée (/10) & 3,17 & 3,20 & 2,96 \\
\hline Flaveur métallique/oxydée (/10) & $1,02(\mathrm{c})$ & 1,66 (b) & 2,27 (a) \\
\hline Flaveur piquante $(/ 10)$ & 1,19 (b) & 1,43 (a) & 1,76 (a) \\
\hline Flaveur rance $(/ 10)$ & $0,31(\mathrm{c})$ & 0,74 (b) & 1,97 (a) \\
\hline Flaveur savon $(/ 10)$ & 0,13 (b) & 0,24 (b) & 1,22 (a) \\
\hline \multicolumn{4}{|l|}{ Composition des fromages } \\
\hline Lipolyse (g AO.100 g-1 MG) & $0,72(\mathrm{c})$ & 1,20 (b) & 2,92 (a) \\
\hline $\mathrm{pH}$ & 4,20 & 4,20 & 4,17 \\
\hline ES (\%) & 40,91 (b) & 43,00 (a) & 42,62 (a) \\
\hline $\mathrm{G} / \mathrm{S}(\%)$ & 47,87 (a) & 48,25 (a) & 41,91 (b) \\
\hline HFD (\%) & 73,50 (a) & 71,92 (b) & $69,93(\mathrm{c})$ \\
\hline$\%$ de fabrications au lait pasteurisé & 30,8 & 56,4 & 50,0 \\
\hline \multicolumn{4}{|l|}{ Composition des laits utilisés } \\
\hline Lipolyse (g AO.100 g $\left.{ }^{-1} \mathrm{MG}\right)$ & $0,20(\mathrm{c})$ & 0,34 (b) & 1,32 (a) \\
\hline $\mathrm{MG}\left(\mathrm{g} \cdot \mathrm{L}^{-1}\right)$ & 29,12 (a) & 28,79 (a) & $25,12(\mathrm{~b})$ \\
\hline $\mathrm{MP}\left(\mathrm{g} \cdot \mathrm{L}^{-1}\right)$ & 29,46 (a) & $28,43(b)$ & $27,34(\mathrm{c})$ \\
\hline $\mathrm{MG} / \mathrm{MP}$ & 0,99 (a) & $1,01(a)$ & 0,92 (b) \\
\hline $\mathrm{pH}$ & $6,72(a)$ & 6,71 (a) & $6,65(b)$ \\
\hline Coliformes totaux $\left(\log \mathrm{UFC} \cdot \mathrm{mL}^{-1}\right)$ & 2,65 & 1,43 & 1,99 \\
\hline Cellules somatiques $\left(\log \mathrm{CCS} \cdot \mathrm{mL}^{-1}\right)$ & 6,08 & 6,04 & 5,99 \\
\hline FMAR $\left(\log \mathrm{UFC} \cdot \mathrm{mL}^{-1}\right)$ & 4,91 & 4,99 & 4,99 \\
\hline Psychrotrophes $\left(\log\right.$ UFC.mL $\left.L^{-1}\right)$ & 4,30 & 3,76 & 4,25 \\
\hline
\end{tabular}

(a) (b) (c) : les moyennes suivies d'une lettre distincte sont significativement différentes $(p<0,05)$.

* : pour les descripteurs de défauts, les chiffres en gras correspondent à des valeurs moyennes supérieures aux valeurs seuils d'apparition de défauts présentées dans le tableau I.

organoleptiques. En revanche, la flaveur chèvre des fromages affinés FA4 de qualité Q2 était en moyenne plus intense que celle des fromages affinés FA4 de qualité Q1.

De manière générale, les notes moyennes attribuées aux descripteurs de défauts étaient plus élevées pour les fromages affinés de la catégorie Q3 que pour les fromages affinés Q1 et Q2. Les principaux défauts organoleptiques des fromages affinés FA8 de qualité Q3 étaient des odeurs rance, bleue et piquante, et des flaveurs amère, 


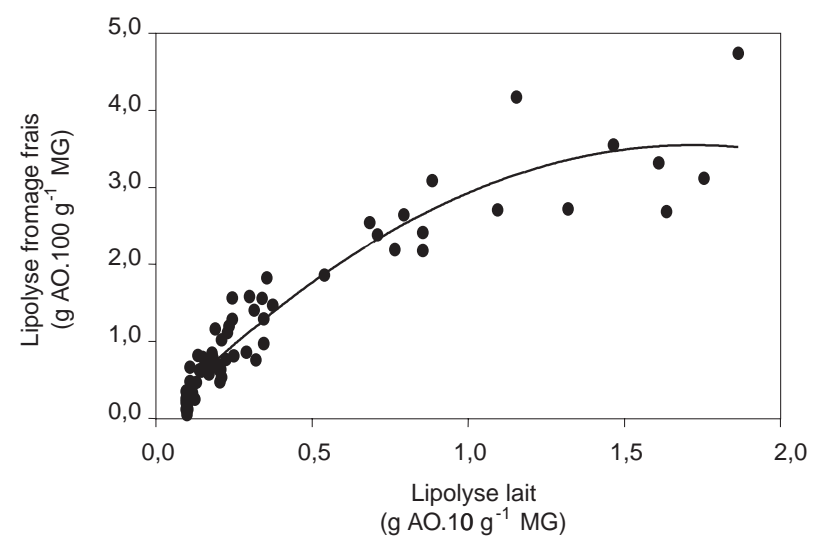

Figure 2. Niveau de lipolyse des fromages frais en fonction du niveau de lipolyse des laits utilisés. Figure 2. Lipolysis level of fresh cheeses as a function of lipolysis level of the milks used.

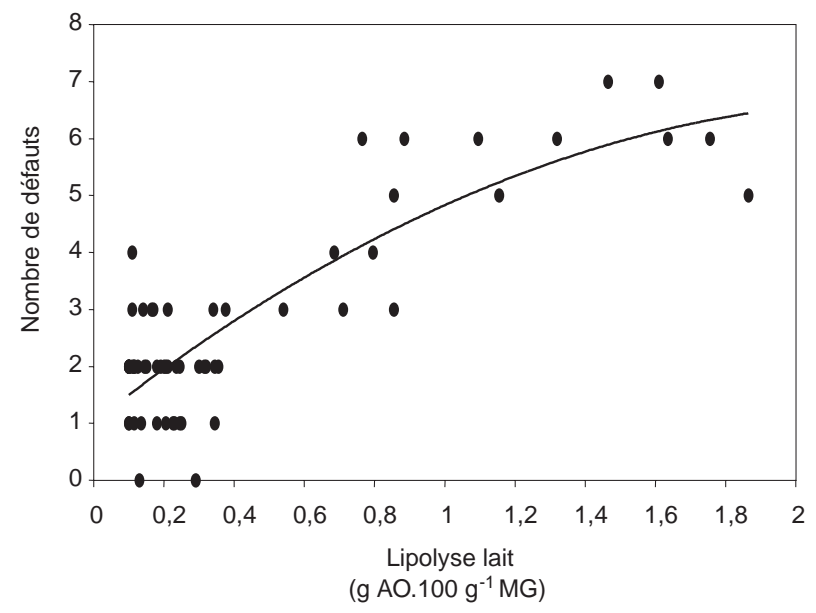

Figure 3. Nombre de défauts organoleptiques perçus sur les fromages frais en fonction du niveau de lipolyse des laits utilisés.

Figure 3. Number of sensorial defects perceived on fresh cheeses as a function of lipolysis level of the milks used.

piquante, rance et savon. Les notes moyennes des descripteurs de défauts des fromages affinés FA8 étaient sensiblement plus élevées que celles des fromages affinés FA4. Le défaut d'amertume était couramment rencontré chez l'ensemble des fromages affinés analysés, mais son intensité moyenne était significativement plus basse pour les fromages Q1 en comparaison aux fromages de qualité Q2 et Q3 (Tab. III).
Les fromages affinés FA4 de qualité Q1 possédaient un niveau de lipolyse significativement plus bas comparativement aux produits de qualité Q2. Des tendances similaires étaient observées pour les fromages affinés FA8 (Tab. III).

Aucun effet du niveau de lipolyse des laits utilisés n'était observé sur la qualité organoleptique des fromages affinés (Tab. III). En revanche, d'autres paramètres, 
comme les teneurs en MG et MP des laits, l'HFD au démoulage et le G/S des fromages semblaient liés à la qualité sensorielle des fromages affinés. Ainsi, comparativement aux produits de qualité Q1 et Q2, les fromages affinés FA8 de qualité Q3

Tableau III. Caractéristiques moyennes des fromages et des laits utilisés en fonction de la qualité sensorielle des fromages affinés.

Table III. Mean characteristics of cheeses and milk used as a function of the sensorial quality of ripened cheeses.

\begin{tabular}{|c|c|c|c|c|c|}
\hline \multirow[b]{2}{*}{ Qualité sensorielle des fromages: } & \multicolumn{2}{|c|}{ Fromages affinés FA4 } & \multicolumn{3}{|c|}{ Fromages affinés FA 8} \\
\hline & $\mathbf{Q}_{1}$ & $\mathbf{Q}_{2}$ & $\mathbf{Q}_{1}$ & $\mathbf{Q}_{2}$ & $\mathbf{Q}_{3}$ \\
\hline & $(n=39)$ & $(n=21)$ & $(n=11)$ & $(n=44)$ & $(n=5)$ \\
\hline \multicolumn{6}{|l|}{ Caractéristiques organoleptiques* } \\
\hline Odeur globale (/10) & 4,06 (a) & 5,24 (a) & $4,16(\mathrm{c})$ & 5,03 (b) & 6,35 (a) \\
\hline Flaveur globale $(/ 10)$ & $5,80(\mathrm{~b})$ & 6,61 (a) & $6,07(\mathrm{c})$ & $6,83(b)$ & 7,45 (a) \\
\hline Flaveur chèvre (/10) & $3,86(b)$ & 4,46 (a) & 3,65 & 4,25 & 4,28 \\
\hline Nombre moyen de défauts (/11) & 0,58 (b) & 2,66 (a) & $0,81(\mathrm{c})$ & 3,27 (b) & 7,20 (a) \\
\hline Odeur rance $(/ 10)$ & 0,13 & 0,24 & $0,06(\mathrm{c})$ & 0,24 (b) & 1,07 (a) \\
\hline Odeur aigre (/10) & $0,30(b)$ & 0,52 (a) & 0,31 & 0,47 & 1,08 \\
\hline Odeur bleue (/10) & $0,56(b)$ & 0,95 (a) & $0,74(\mathrm{c})$ & $1,43(b)$ & 2,60 (a) \\
\hline Odeur piquante $(/ 10)$ & 0,64 (b) & 1,13 (a) & $1,01(\mathrm{c})$ & $1,41(\mathrm{c})$ & 3,01 (a) \\
\hline Flaveur acide (/10) & $1,03(b)$ & 1,32 (a) & 0,79 (b) & 1,10 (a) & $1,16(a)$ \\
\hline Flaveur amère $(/ 10)$ & 2,04 (b) & 2,86 (a) & 2,09 (c) & 3,12 (b) & 4,01 (a) \\
\hline Flaveur salée (/10) & 3,43 (b) & 4,06 (a) & 3,58 (b) & 4,25 (a) & 4,51 (a) \\
\hline Flaveur métallique/oxydée (/10) & 0,66 (b) & 1,09 (a) & 0,56 & 0,85 & 1,10 \\
\hline Flaveur piquante $(/ 10)$ & 1,42 (b) & 2,61 (a) & 2,25 (b) & 3,83 (a) & 4,42 (a) \\
\hline Flaveur rance $(/ 10)$ & 0,54 (b) & 0,96 (a) & $0,21(\mathrm{c})$ & 0,83 (b) & 1,87 (a) \\
\hline Flaveur savon (/10) & 0,58 (b) & 1,23 (a) & 0,66 (b) & 1,52 (a) & 2,38 (a) \\
\hline \multicolumn{6}{|l|}{ Composition des fromages } \\
\hline Lipolyse (g AO.100 g $\left.{ }^{-1} \cdot \mathrm{MG}\right)$ & $7,37(b)$ & 9,68 (a) & 9,99 (b) & $12,24(\mathrm{a})$ & $10,90(\mathrm{ab})$ \\
\hline $\mathrm{pH}$ & 5,76 (b) & 5,91 (a) & $6,41(b)$ & 6,57 (a) & 6,31 (b) \\
\hline ES (\%) & 48,98 (a) & 46,63 (b) & 49,97 & 48,63 & 49,35 \\
\hline $\mathrm{G} / \mathrm{S}(\%)$ & 49,85 & 50,76 & $50,70(b)$ & 50,31 (b) & 53,16 (a) \\
\hline HFD (\%) & 67,47 (b) & 69,90 (a) & 66,98 & 67,98 & 68,60 \\
\hline HFD au démoulage (\%) & 73,08 & 73,81 & 72,67 (b) & 73,24 (b) & 75,66 (a) \\
\hline$\%$ de fabrications au lait pasteurisé & 66,7 (a) & 19,0 (b) & 72,7 (a) & $47,7(\mathrm{ab})$ & 20,0 (b) \\
\hline \multicolumn{6}{|l|}{ Composition des laits utilisés } \\
\hline Lipolyse $\left(\mathrm{g} \mathrm{AO} .100 \mathrm{~g}^{-1} \cdot \mathrm{MG}\right)$ & 0,50 & 0,37 & 0,42 (a) & 0,48 (a) & 0,19 (b) \\
\hline MG $\left(g \cdot L^{-1}\right)$ & 27,49 (b) & 30,01 (a) & 27,26 (b) & 28,02 (b) & 33,91 (a) \\
\hline $\mathrm{MP}\left(\mathrm{g} \cdot \mathrm{L}^{-1}\right)$ & 27,93 (b) & 29,58 (a) & 28,33 (b) & 28,36 (b) & 30,21 (a) \\
\hline ratio $\mathrm{MG} / \mathrm{MP}$ & 0,98 & 1,01 & 0,96 (b) & 0,99 (b) & 1,12 (a) \\
\hline $\mathrm{pH}$ & 6,71 (a) & 6,68 (b) & 6,73 (a) & 6,69 (b) & $6,72(a b)$ \\
\hline Coliformes totaux $\left(\log \mathrm{UFC} \cdot \mathrm{mL}^{-1}\right)$ & 1,18 (b) & 2,46 (a) & $0,01(\mathrm{c})$ & 1,75 (b) & 3,38 (a) \\
\hline Cellules somatiques $\left(\log \mathrm{CCS} \cdot \mathrm{mL}^{-1}\right)$ & 6,02 (b) & 6,09 (a) & $6,06(a b)$ & 6,03 (b) & 6,09 (a) \\
\hline FMAR $\left(\log\right.$ UFC $\left.\cdot \mathrm{mL}^{-1}\right)$ & 3,23 (b) & 4,72 (a) & $2,04(\mathrm{c})$ & 3,87 (b) & 5,85 (a) \\
\hline Psychrotrophes $\left(\log \mathrm{UFC} \cdot \mathrm{mL}^{-1}\right)$ & 4,87 (b) & 5,15 (a) & 4,89 & 4,97 & 5,15 \\
\hline
\end{tabular}

(a) (b) (c) (ab) : les moyennes suivies d'une lettre distincte sont significativement différentes $(p<0,05)$.

* : pour les descripteurs de défauts, les chiffres en gras correspondent à des valeurs moyennes supérieures ou égales aux valeurs seuils d'apparition de défauts présentées dans le tableau I. 
possédaient un G/S et un HFD au démoulage significativement plus élevés (respectivement 53,2\% et 75,7 \%), lié à la mise en œuvre de laits présentant un ratio MG/MP supérieur à 1,1 (Tab. III).

Les résultats du tableau III indiquent également que les laits utilisés pour l'obtention de fromages affinés de qualité satisfaisante étaient de meilleure qualité microbiologique que les laits conduisant à des fromages de qualité Q2 ou Q3. Ces résultats peuvent être mis en relation avec le pourcentage de fabrications au lait pasteurisé, qui était significativement plus élevé pour les fromages de qualité satisfaisante (Tab. III).

\subsection{Effet de la pasteurisation}

La pasteurisation du lait entraînait une diminution significative du niveau de lipolyse des fromages (Fig. 4).

Aucun effet significatif de la pasteurisation n'était observé sur la qualité sensorielle des fromages frais (Tab. II). Pour les fromages affinés en revanche, la part des fabrications au lait pasteurisé était nettement majoritaire dans les produits de qualité Q1
(Tab. III). Les caractéristiques sensorielles moyennes des fromages affinés fabriqués au lait cru ou au lait pasteurisé sont indiquées dans le tableau IV. De manière générale, l'intensité des descripteurs sensoriels et le nombre de défauts étaient plus bas pour les fromages au lait pasteurisé comparativement aux fromages au lait cru. En particulier, les intensités moyennes des défauts rance savon et piquant étaient significativement plus basses pour les produits au lait pasteurisé. L'odeur et la flaveur globales des fromages au lait pasteurisé étaient moins intenses que celles des fromages au lait cru mais aucune différence significative n'était observée concernant la flaveur chèvre (Tab. IV).

Ces résultats peuvent être mis en relation notamment avec la qualité microbiologique des laits utilisés pour la fabrication de fromages affinés de qualité satisfaisante (Tab. III). Le nombre de germes totaux, de coliformes totaux et, dans une moindre mesure, de germes psychrotrophes était significativement moins élevé dans les laits conduisant à l'obtention de fromages affinés de qualité Q1. Par ailleurs, l'HFD moyen des produits fabriqués à partir de lait

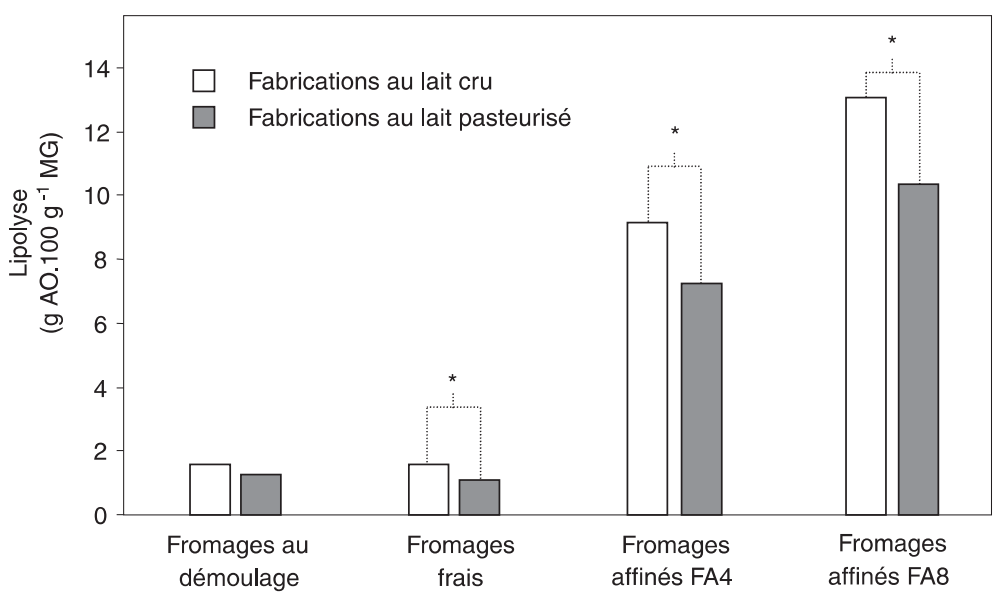

Figure 4. Effet de la pasteurisation sur le niveau de lipolyse des laits et des fromages $(*$ : différence significative, $p<0,05$ ).

Figure 4. Effect of pasteurisation on the lipolysis level of milk and cheeses $(*$ : significant difference, $p<0.05)$. 
Tableau IV. Caractéristiques sensorielles moyennes des fromages affinés fabriqués au lait cru ou pasteurisé.

Table IV. Mean sensorial characteristics of ripened cheeses made from raw or pasteurised milk.

\begin{tabular}{lcc|cc}
\hline & \multicolumn{2}{c|}{ Fromages affinés FA4 } & \multicolumn{2}{c}{ Fromages affinés FA8 } \\
\hline & Lait cru & Lait past. & Lait cru & Lait past. \\
\hline Caractéristiques organoleptiques & $(n=30)$ & $(n=30)$ & $(n=30)$ & $(n=30)$ \\
\hline Odeur globale $(/ 10)$ & $4,90(\mathrm{a})$ & $4,06(\mathrm{~b})$ & $5,28(\mathrm{a})$ & $4,67(\mathrm{~b})$ \\
Flaveur globale $(/ 10)$ & $6,24(\mathrm{a})$ & $5,92(\mathrm{~b})$ & $6,94(\mathrm{a})$ & $6,56(\mathrm{~b})$ \\
Flaveur chèvre $(/ 10)$ & 4,14 & 4,00 & 4,08 & 4,21 \\
Nombre moyen de défauts $(/ 11)$ & $1,80(\mathrm{a})$ & $0,83(\mathrm{~b})$ & $3,57(\mathrm{a})$ & $2,73(\mathrm{~b})$ \\
Odeur rance $(/ 10)$ & $0,23(\mathrm{a})$ & $0,10(\mathrm{~b})$ & 0,30 & 0,26 \\
Odeur aigre $(/ 10)$ & $0,46(\mathrm{a})$ & $0,30(\mathrm{~b})$ & 0,53 & 0,45 \\
Odeur bleue $(/ 10)$ & $0,80(\mathrm{a})$ & $0,59(\mathrm{~b})$ & 1,49 & 1,32 \\
Odeur piquante $(/ 10)$ & 0,89 & 0,74 & 1,61 & 1,33 \\
Flaveur acide $(/ 10)$ & 1,17 & 1,09 & $1,16(\mathrm{a})$ & $0,94(\mathrm{~b})$ \\
Flaveur amère $(/ 10)$ & $\mathbf{2 , 4 3}$ & $\mathbf{2 , 2 3}$ & $\mathbf{3 , 0 6}$ & $\mathbf{2 , 9 6}$ \\
Flaveur salée $(/ 10)$ & 3,75 & 3,56 & 4,26 & 4,05 \\
Flaveur métallique/oxydée $(/ 10)$ & 0,85 & 0,78 & 0,87 & 0,76 \\
Flaveur piquante $(/ 10)$ & $2,18(\mathrm{a})$ & $1,50(\mathrm{~b})$ & $\mathbf{4 , 1 6}(\mathbf{a})$ & $\mathbf{3 , 0 2}$ (b) \\
Flaveur rance $(/ 10)$ & $0,83(\mathrm{a})$ & $0,55(\mathrm{~b})$ & $0,94(\mathrm{a})$ & $0,67(\mathrm{~b})$ \\
Flaveur savon $(/ 10)$ & $1,00(\mathrm{a})$ & $0,62(\mathrm{~b})$ & $\mathbf{1 , 6 4}(\mathbf{a})$ & $1,23(\mathrm{~b})$ \\
\hline
\end{tabular}

(a) (b) : les moyennes suivies d'une lettre distincte sont significativement différentes $(p<0,05)$.

* : pour les descripteurs de défauts, les chiffres en gras correspondent à des valeurs moyennes supérieures aux valeurs seuils d'apparition de défauts présentées dans le tableau I.

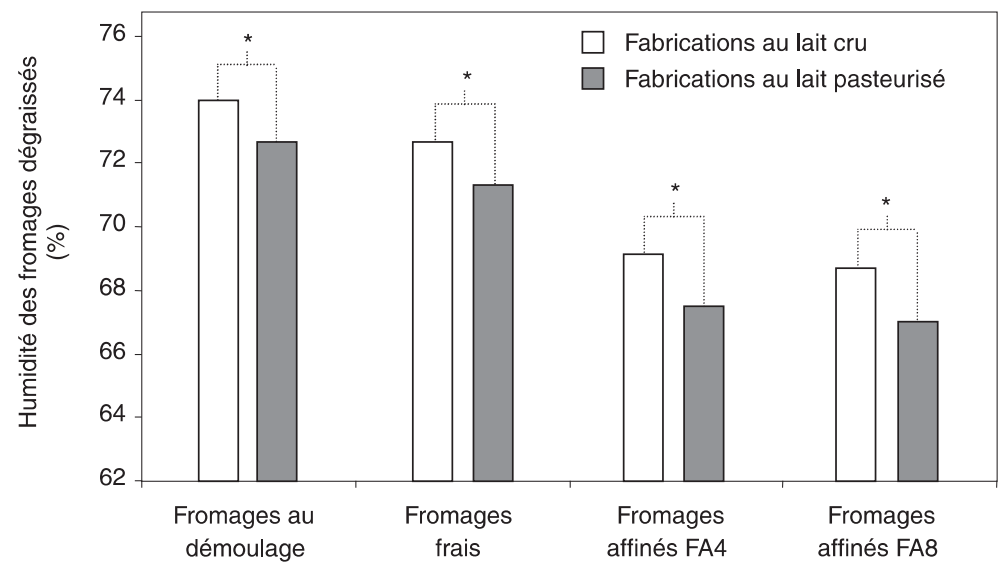

Figure 5. Effet de la pasteurisation du lait sur l'humidité des fromages dégraissés $(*$ : différence significative, $p<0,05)$.

Figure 5. Effect of milk pasteurisation on moisture in non-fat cheeses $(*$ : significant difference, $p<0.05)$. 
pasteurisé était significativement plus faible au démoulage et le restait pendant toute la durée de vie des produits (Fig. 5).

\section{DISCUSSION}

Les résultats présentés indiquent que la qualité sensorielle des fromages frais était corrélée négativement au niveau de lipolyse du lait utilisé. L'emploi de laits fortement lipolysés conduisait à l'obtention de fromages frais présentant également des forts niveaux de lipolyse (Fig. 2). La mise en œuvre de laits dont le niveau de lipolyse était inférieur à $0.4 \mathrm{~g} \mathrm{AO} \cdot 100 \mathrm{~g}^{-1} \mathrm{MG}$ conduisait à l'obtention de fromages frais possédant 3 défauts au plus (sauf 1 produit qui présentait 4 défauts), alors que l'utilisation de laits dont le niveau de lipolyse excédait $1 \mathrm{~g} \mathrm{AO} \cdot 100 \mathrm{~g}^{-1} \mathrm{MG}$ conduisait à l'obtention de fromages frais possédant au moins 5 défauts (Fig. 3). Les défauts perçus sur ces fromages (rance, savon, piquant, cf. Tab. II) peuvent être liés à des quantités excessives d'acides gras à chaînes courtes et moyennes [3, 32]. Ces acides gras en excès pourraient provenir de l'activité enzymatique de la LPL. Bien que le $\mathrm{pH}$ des fromages frais $(\sim \mathrm{pH} 4,2)$ ne correspondait pas au pH optimum de la LPL ( $\mathrm{pH}$ neutre ou alcalin selon Olivecrona, [29]), une activité lipasique résiduelle de la LPL restait possible avant et pendant l'acidification, qui est un processus lent en technologie lactique (20 à $24 \mathrm{~h}$ ). Les autres lipases éventuellement actives dans les fromages proviennent des bactéries et des cellules somatiques présentes dans le lait, des ferments lactiques, de la flore d'affinage et de la présure $[6,16]$. Dans cette étude, quelle que soit la qualité sensorielle des fromages frais, les comptages de cellules somatiques et la population en germes psychrotrophes des laits mis en œuvre étaient comparables. De plus, l'activité lipolytique très faible des ferments lactiques commerciaux pouvait être négligée et aucune flore d'affinage n'était utilisée pour la fabrication des fromages frais.
En revanche, nous n'avons pas caractérisé l'action lipasique potentielle de la présure employée pour les fabrications, qui peut provenir de la présence d'une certaine quantité de lipase prégastrique de veau. Par ailleurs, un défaut de flaveur métallique/oxydée était souvent observé chez les fromages frais, ce qui pourrait être dû à la dégradation oxydative de la matière grasse [28].

La qualité sensorielle des fromages affinés n'était pas liée au niveau de lipolyse des laits mis en œuvre (compris dans cette étude entre 0,1 et $1,9 \mathrm{~g}$ AO. $100 \mathrm{~g}^{-1} \mathrm{MG}$ ) mais aux paramètres technologiques, et plus particulièrement à l'HFD des fromages au démoulage. Le risque d'obtenir des fromages affinés de qualité organoleptique inacceptable était plus important pour des niveaux d'HFD au démoulage supérieurs à $75 \%$, il était par contre faible pour des niveaux d'HFD au démoulage inférieurs à $73 \%$. En effet, plus l'HFD au démoulage est élevée, plus la disponibilité de l'eau est importante, ce qui peut favoriser les activités microbiennes et enzymatiques [26], et, par conséquent, conduire à un mauvais contrôle de l'affinage et à l'apparition de défauts de flaveur. En particulier, les défauts d'odeur bleue et piquante, ainsi que les défauts de flaveur piquante, rance et savon (Tab. III) pouvaient être liés à un développement plus important de la flore de surface. Par ailleurs, un défaut d'amertume était souvent observé chez les fromages affinés (Tab. III), qui pourrait être dû à des quantités importantes de mono et diglycérides, à la présence de peptides hydrophobes issus de la protéolyse [24] et à un effet des sels tels que $\mathrm{CaCl}_{2}$ et $\mathrm{MgCl}_{2}$ [9].

Nos résultats indiquent également que la mise en œuvre de laits présentant des ratios MG/MP supérieurs à 1,1 pouvait conduire à l'obtention de fromages à défauts comme dans le cas des fromages affinés FA8 de qualité Q3 (Tab. III). Dans ce cas, un contrôle de l'HFD au démoulage devrait permettre de réduire le risque d'apparition des défauts sensoriels. 
La flaveur caractéristique "chèvre» n'était pas influencée de manière significative par le niveau de lipolyse des laits mis en œuvre, sauf dans le cas des fromages affinés dégustés après 4 semaines de conservation (Tab. III).

Enfin, les résultats présentés montrent que la pasteurisation du lait conduisait à une diminution du niveau de lipolyse des fromages de chèvre (Fig. 4). Des résultats identiques ont été obtenus sur d'autres types de fromages $[4,18]$. La réduction du niveau de lipolyse des fromages frais fabriqués à partir de lait pasteurisé n'était pas accompagné d'une réduction de la fréquence d'apparition des défauts. En revanche, la pasteurisation du lait destiné aux fabrications de fromages affinés conduisait à une diminution de l'intensité et du nombre de défauts organoleptiques (Tab. III). La pasteurisation permet l'inactivation de la LPL [21] et la standardisation microbiologique des laits. La pasteurisation agit également sur l'étape d'égouttage des caillés : l'égouttage était plus rapide pour les fabrications au lait pasteurisé comparativement aux fabrications au lait cru ( 1 à $2 \mathrm{~h}$ de moins pour le même volume de sérum exsudé). De plus, l'extrait sec des sérums était significativement plus élevé pour les fabrications au lait cru que pour les fabrications au lait pasteurisé $\left(58,3 \mathrm{~g} \cdot \mathrm{kg}^{-1}\right.$ contre $56,4 \mathrm{~g} \cdot \mathrm{kg}^{-1}$ respectivement, $p<0,001$ ). Pour un volume de sérum exsudé identique, l'extrait sec des fromages au lait pasteurisé était donc supérieur à celui des fromages au lait cru. Ces différences se répercutaient sur l'HFD des fromages, plus bas dans le cas des fabrications au lait pasteurisé (Fig. 5), ce qui permettait un développement mieux contrôlé de la flore de surface au cours de l'affinage [26]. Ces différentes modifications pourraient expliquer l'effet bénéfique de la pasteurisation sur l'apparition des défauts organoleptiques des fromages affinés. Il convient de préciser toutefois que l'emploi de laits pasteurisés entraînait également une diminution de l'odeur globale et de la flaveur globale des fromages affinés
(Tab. IV), et réduisait de manière non spécifique l'expression aromatique de ces produits. Ces résultats sont en accord avec de nombreux autres travaux qui ont montré que les fromages fabriqués au lait pasteurisé ont une flaveur moins prononcée que ceux fabriqués au lait cru [18].

Ainsi, la maîtrise technologique de la qualité sensorielle des fromages de chèvre demande la mise en œuvre de moyens adaptés au cas des fromages frais et à celui des fromages affinés.

Pour les fromages frais, des seuils du niveau de lipolyse du lait peuvent être proposés afin de maîtriser la qualité sensorielle des produits : le risque d'obtenir des produits de qualité organoleptique inacceptable est faible si le niveau de lipolyse des laits est inférieur à $0,4 \mathrm{~g} \mathrm{AO} \cdot 100 \mathrm{~g}^{-1} \mathrm{MG}$, et ce risque est important pour des niveaux de lipolyse des laits supérieurs à $1 \mathrm{~g}$ AO $100 \mathrm{~g}^{-1} \mathrm{MG}$.

Pour les fromages affinés, le contrôle de la qualité sensorielle des fromages affinés nécessite des travaux supplémentaires, qui privilégient une approche multi-critères intégrant des facteurs liés à la composition du lait (ratio MG/MP) et aux paramètres technologiques (standardisation en $\mathrm{MG}$, contrôle de l'HFD au démoulage, maîtrise de l'affinage).

\section{REMERCIEMENTS}

Les auteurs remercient V. Bonnin pour les analyses physico-chimiques et microbiologiques et $\mathrm{C}$. Duchesne pour les analyses sensorielles. $\mathrm{Ce}$ travail a été financé en partie par l'Union Européenne dans le cadre du programme $\mathrm{n}^{\circ}$ FAIR - CT 98 - 9598.

\section{RÉFÉRENCES}

[1] AFNOR, Guide pour l'implantation d'un local destiné aux analyses sensorielles, Norme NF V09-105 (1972).

[2] Björke K., Castberg H.B., Lipolytic activity in goat's milk, Nordeuropaiesk Mejeri-Tidsskrift 42 (1976) 296-304. 
[3] Brennand C.P., Ha J.K., Lindsay R.C., Aroma properties and threshold of some branchedchain and other minor volatile fatty acids occurring in milkfat and meat lipids, J. Sens. Studies 4 (1989) 105-120.

[4] Chavarri F., Bustamante M.A., Santisteban A., Virto M., Albisu M., Barron L.J.R., de Renobales M., Effect of milk pasteurization on lipolysis during ripening of ovine cheese manufactured at different times of the year, Lait 80 (2000) 433-444.

[5] Chilliard Y., Variations physiologiques des activités lipasiques et de la lipolyse spontanée dans les laits de vache, de chèvre et de femme, Lait 62 (1982) 1-31; 126-154.

[6] Chilliard Y., Lamberet G., La lipolyse dans le lait : les différents types, mécanismes, facteurs de variation, signification pratique, Lait 64 (1984) 544-578.

[7] Chilliard Y., Selselet-Attou G., Bas P., MorandFehr P., Characteristics of lipolytic system in goat milk, J. Dairy Sci. 67 (1984) 2216-2223.

[8] Delacroix-Buchet A, Degas C., Lamberet G., Vassal L., Influence des variants AA et FF de la caséine $\alpha_{S 1}$ caprine sur le rendement fromager et les caractéristiques sensorielles des fromages, Lait 76 (1996) 217-241.

[9] Engel E., Nicklaus S., Septier C., Salles C., Le Quéré J. L., Taste active compounds in a goat cheese water-soluble extract. 2. Determination of the relative impact of water-soluble extract components on its taste using omission tests, J. Agric. Food Chem. 48 (2000) 4260-4267.

[10] FIL, Dénombrement des coliformes, Norme FIL/IDF 73A, Int. Dairy Fed., Brussels, Belgium, 1985.

[11] FIL, Dénombrement des microorganismes psychrotrophes, Norme FIL/IDF 101A, Int. Dairy Fed., Brussels, Belgium, 1991.

[12] FIL, Numération des cellules somatiques du lait, Norme FIL/IDF 148A, Int. Dairy Fed., Brussels, Belgium, 1995.

[13] FIL, Détermination des teneurs en matière grasse laitière, protéines et lactose, Norme FIL/IDF 141B, Int. Dairy Fed., Brussels, Belgium, 1996.

[14] FIL, Determination of free fatty acids in milk and milk products, Bull. Int. Dairy Fed. 265 (1991) $2-51$.

[15] FIL, Methods for assessing the bacteriological quality of raw milk from the farm, Bull. Int. Dairy Fed. 256 (1991) 2-62.

[16] Fox P.F., Law J., McSweeney P.L.H., Wallace J., Biochemistry of cheese ripening, in: Fox P.F. (Ed.), Cheese : Chemistry, physics and microbiology, vol. 1, Chapaman \& Hall, London, UK, 1993, pp. 389-438.

[17] Gaborit P., Ménard A., Morgan F., Impact of ripening strains on the typical flavour of goat cheeses, Int. Dairy J. (2001) 315-325.
[18] Grappin R., Beuvier E., Review : Possible implications of milk pasteurization on the manufacture and sensory quality of ripened cheese, Int. Dairy J. (1997) 7, 751-761.

[19] Ha J.K., Lindsay R.C., Contributions of cow, sheep, and goat milks to characterizing branched-chain fatty acid and phenolic flavors in varietal cheeses, J. Dairy Sci. 74 (1991) 3267-3274.

[20] Heiss E., Studies on the determination of fat matter in cheeses by rapid methods, Dtschl. Milchztg. 82 (1961) 3.

[21] Jandal J.M., Some factors affecting lipase activity in goat milk, Small Rumin. Res. 16 (1995) 87-91.

[22] Lamberet G., Degas C., Delacroix-Buchet A., Vassal L., Influence de caractères liés aux allèles A et $\mathrm{F}$ de la caséine $\alpha_{\mathrm{S} 1}$ caprine sur la flaveur chèvre : fabrications fromagères avec échange de protéines et de matière grasses, Lait 76 (1996) 349-361.

[23] Le Mens P., Heuchel V., Jaubert G., Bodin J.P., Sauvageot F., Humbert G., Caractérisation et origine des défauts de flaveur dans les fromages de chèvre. Compte rendu de fin d'étude no 9983110 - Prog. Aliment 2000, Institut de l'Élevage, Ed. Technipel, Paris, 1997, 70 p.

[24] Lemieux L., Simard R.E., Bitter flavour in dairy products. I. A review of the factors likely to influence its development, maily in cheese mahufacture, Lait 71 (1991) 599-636.

[25] Le Quéré J.L., Pierre A., Riaublanc A., Demaizières D., Characterization of aroma compounds in the volatile fraction of soft goat cheese during ripening, Lait 78 (1998) 279-290.

[26] Mahaut M., Jeantet R., Brulé G., Initiation à la technologie fromagère, Éditions Tec \& Doc, Paris, France, 2000.

[27] Morgan F., Gaborit P., The typical flavour of goat milk products: technological aspects, Int. J. Dairy Technol. 54 (2001) 38-40.

[28] Nicholson J.W.G., Spontaneous oxidised flavor in cow's milk, Bull. Int. Dairy Fed. 281 (1993) 2-12.

[29] Olivecrona T., Aspects biochimiques de la lipolyse du lait de vache, Bull. Int. Dairy Fed. 118 (1980) 20-27.

[30] Pierre A., Le Quéré J.-L., Famelart M.-H., Riaublanc A., Rousseau F., Composition, yield, texture and aroma compounds of goat cheeses as related to the $A$ and $O$ variants of $\alpha_{S 1}$ casein in milk, Lait 78 (1998) 291-301.

[31] Skjevdal T., Flavour of goat's milk: a review of studies on the sources of its variation, Livest. Product. Sci. 6 (1979) 397-405.

[32] Woo A.H., Lindsay R.C., Concentration of major free fatty acids and flavour development in Italian cheese varieties, J. Dairy Sci. 67 (1984) 960-968. 